\title{
Le tombeau vide pour Shakespeare et Chapman : variations autour d'un signe théâtral
}

Lætitia Coussement-Boillot et Christine Sukic

\section{(2) OpenEdition}

\section{Journals}

Édition électronique

URL : http://journals.openedition.org/shakespeare/783

DOI : 10.4000/shakespeare.783

ISSN : 2271-6424

Éditeur

Société Française Shakespeare

Édition imprimée

Date de publication : 1 novembre 2002

Pagination : 85-101

Référence électronique

Lætitia Coussement-Boillot et Christine Sukic, « Le tombeau vide pour Shakespeare et Chapman variations autour d'un signe théâtral », Actes des congrès de la Société française Shakespeare [En ligne], 20 | 2002, mis en ligne le 01 novembre 2007, consulté le 01 mai 2019. URL : http:// journals.openedition.org/shakespeare/783 ; DOI : 10.4000/shakespeare.783 


\section{S H A K E S P E A R E \& S E S \\ C O N T E M P O R A I N S}

Société Française Shakespeare

Actes du Congrès de 2002

$* * *$

Textes réunis et présentés par

Patricia DORVAL

publiés sous la direction de

Jean-Marie MAGUIN 


\section{E T O M B E A U V IDE POUR SHA K ES PEA RE ET CHAPMA N : VARIATIONSAUTOUR D'UN SIGNE T H É Â T R A L}

Le tombeau vide, signe théâtral primordial dans les représentations médiévales de. la Passion du Christ ', trouve peut-être son origine dans la présence d'imitations du Saint-Sépulcre dans les premières églises chrétiennes ${ }^{2}$. Dans les parties de ces cycles de pièces mettant en scène la Résurrection, le contenant vide qu'était la sépulture du Christ se trouvait au centre du lieu de la représentation ${ }^{3}$. On retrouve, bien sûr, l'image de ce tombeau vide dans la poésie religieuse des XVIe et XVIIe siècles en Angleterre. Là, comme dans les représentations médiévales, la découverte du tombeau vide donne lieu à des lamentations de la part des «trois Marie» - la Vierge Marie, Marie-Madeleine et Marie, mère de Jacques - mais ce moment de désespoir est de courte durée, puisqu'il est suivi par l'annonce de la résurrection ". Néanmoins, la découverte du tombeau vide montre aussi qu'il y a décalage entre l'insupportable apparence l'absence du corps - et la réalité que masque cette apparence : la résurrection. Dans les cycles médiévaux, les soldats chargés de veiller sur le sépulcre étaient d'ailleurs frappés de stupeur au moment où le Christ quittait le tombeau, comme pour montrer l'éblouissement des sens face à cet événement hors du commun. Ce décalage entre apparence et réalité 
autour d'un tombeau vide se rencontre dans deux pièces de la première moitié du XVIIe siècle presque contemporaines, The Widow's Tears de George Chapman, comédie publiée en 1612 mais sans doute composée vers $1605-06^{s}$, et Pericles, pièce romanesque écrite par Shakespeare probablement en 1607 - en collaboration hypothétique avec d'autres dramaturges. Néanmoins, l'usage qui est fait de ce motif par les deux dramaturges se détache de son modèle médiéval. De plus, Chapman et Shakespeare n'en ont pas la même approche, tant par le style que par la signification. Si, pour Chapman, le motif est prétexte à écrire l'une de ses comédies les plus noires et les plus satiriques, en revanche, Shakespeare, comme souvent, ne se cantonne pas à une interprétation unique et figée. Les variations autour du contenant vide ou dépourvu de son contenu originel permettent au dramaturge d'interroger la nature de l'homme et du monde et d'entraîner le spectateur dans un questionnement vertigineux mais sans réponse sur ce qui est et ce qui devrait être, l'illusion et la vérité.

$$
* * *
$$

Dans The Widow's Tears, Chapman exploite le thème de l'inconstance féminine à travers deux intrigues, liées par leur thématique - la fidélité d'une veuve à son défunt mari. Le personnage principal, Tharsalio, est le grand ordonnateur de cette comédie. La pièce se déroule à Chypre, et l'intrigue principale est tirée de l'épisode de la Matrone d'Éphèse, dans le Satyricon de Pétrone, avec quelques variantes. On y voit Cynthia, la belle-sour de Tharsalio, pleurer son mari Lysander qu'elle croit mort - il veut ainsi mettre sa fidélité à l'épreuve, avec la complicité de Tharsalio - dans un sépulcre qui est vide, comme le fait remarquer un des soldats qui montent la garde autour du tombeau : «For the corpse, sir? Bare misprision, there's no body, nothing. A mere blandation, a deceptio visus» (V.1.542-3) ${ }^{6}$. Presque toute l'action des actes IV et $\mathrm{V}$ de cette pièce se déroule autour de cette forme vide et en son intérieur. Elle est l'emblème de l'inconstance de Cynthia et de l'hypocrisie de ses larmes. Bien sûr, on sait que le motif du tombeau vide dans cette pièce a pour source principale le Satyricon, et il n'est pas question ici de démontrer que Chapman se serait inspiré de manière directe du théâtre religieux du. Moyen Âge. En revanche, on peut dire qu'il exploite un signe dramatique préexistant, notamment dans sa représentation. D'après Akihiro Yamada, le sépulcre, dans la pièce de Chapman, était vraisemblablement représenté par un espace fermé, aménagé contre la paroi des coulisses dans le fond de 
la scène, et ássez grand pour contenir un cercueil - vide, bien sûr - de même que Cynthia et sa suivante Ero'. D'après les indications scéniques données dans la première édition de la pièce, les acteurs doivent pouvoir pénétrer dans cet espace, puisqu'il est dit clairement que Tharsalio frappe à une porte. Quoi qu'il en soit, le tombeau constitue le lieu le plus important de la scène, là où tous les regards convergent, tout comme le tombeau du Christ - sur le plan de la mise en scène — dans les Résurrections médiévales.

Néanmoins, pour Chapman, cet espace vide a une autre signification puisqu'il est emblématique de l'hypocrisie de Cynthia. Mais, loin de s'éloigner du texte de l'Évangile en exploitant ce thème, le poète y revient de manière détournée. Après que Cynthia a accepté les avances de Lysander déguisé en soldat, son frère Tharsalio lui apprend la vérité, et lui annonce la résurrection prochaine de son mari : «That you see yonder is but a shadow, an empty chest containing nothing but air» (V.1.317-9). Tharsalio - grand dénonciateur des fausses apparences dans la pièce presse sa sœur de cesser ses lamentations hypocrites, en mettant en évidence la différence entre l'extérieur de la tombe et son intérieur : «Paint not your tomb without; we know too well / What rotten carcasses are lodg'd within» (V.1.298-9). Le thème de l'hypocrisie rappelle assurément l'image des «sépulcres blanchis», expression utilisée par le Christ dans Mathieu, 23: 27 pour dénoncer l'hypocrisie des Pharisiens. L'image est courante dans la littérature de l'époque. On la trouve par exemple dans la pièce de Thomas Heywood, Edward IV, Part Two (1599) à propos de Charles de Bourgogne: «Charles that painted sepulchre, / And most disloyall Duke of Burgundie» (vers 785-6).

Malgré cette référence indirecte au texte de l'Évangile, la présence du sépulcre au centre de la scène n'annonce pas, pour Chapman, une résurrection à venir, mais est le signe d'un vide irrémédiable qui se cache au cœur des apparences. Ce thème, primordial dans la pièce, n'est pas surprenant pour un auteur tel que lui, puisqu'on connaît sa réputation d'idéologue et de poète pessimiste. Le sépulcre vide est d'ailleurs un motif que l'on retrouve dans d'autres pièces de Chapman, comme dans ses poèmes, où il figure aussi l'absence de vertu et la corruption des valeurs morales. Ainsi, dans sa tragédie The Revenge of Bussy D'Ambois (1611) le dramaturge parle de sépulcres de noblesse, «the breathing Sepulchres of Noblesse» (II.1.154), c'est-à-dire des hommes qui n'ont que les signes extérieurs de la noblesse, mais n'en ont pas la vertu intrinsèque ${ }^{8}$. De même, dans The Tragedy of Byron (1608), le héros, avant son exécution, 
prend soudain conscience de la fragilité de son apparence humaine, aussi fragile que l'air, et qu'un souffle pourrait briser :

I know this body but a sink of folly, The ground-work and raised frame of woe and frailty ;

The bond and bundle of corruption ;

A quick corse, only sensible of grief ;

A walking sepulchre, or household thief,

A glass of air, broken with less than breath.

(V.4.32-7)

Celui qui pensait être le sauveur de la France et l'Atlas soutenant son pays sur ses épaules a soudain conscience de n'être qu'une forme vide au moment de la mort. Comme on le sait, le corps comme sépulcre ou prison de l'âme est une image assez courante'. Chapman l'utilise par exemple dans Casar and Pompey (publiée en 1639), où l'âme, par la mort, peut enfin se séparer de ce sépulcre qu'est le corps, "sever'd from this breathing sepulchre» (III.1.181). Comme dans les autres pièces de Chapman, on trouve dans The Widow's Tears de nombreuses images de contenants vides qui dénoncent l'absence de substance au profit de l'accumulation de formes trompeuses. Ainsi, le motif du sépulcre vide, autour duquel presque toute l'action des deux derniers actes s'agence, est décliné tout au long de la pièce et devient donc le centre névralgique de l'espace dramatique.

Cela est particulièrement évident dans l'autre intrigue de la pièce, qui annonce l'intrigue principale, notamment par des références précises au texte de Pétrone, même si l'histoire de la Matrone d'Éphèse n'est exploitée que plus tard. Dans les trois premiers actes, Tharsalio séduit une jeune veuve, la comtesse Eudora, qui a juré de ne pas se remarier et de demeurer chaste dans son veuvage. Pour cela, il pique son intérêt pour lui en lui faisant dire par une entremetteuse, Arsace, qu'il est l'un des hommes les plus débauchés de l'île. Les dehors vertueux de la comtesse dissimulent un désir secret :

And yet this virtuous Countess hath to my ear spun out many a tedious lecture of pure sister's thread against concupiscence. But ever with such an affected zeal, as my mind gave me, she had a kind of secret titillation. (II.3.17-21) 
On le voit, Chapman en profite pour dénoncer l'hypocrisie puritaine, comme le laisse penser l'utilisation des expressions : «tedious lecture», «pure sister», ou encore «affected zeal». La comtesse apparaît en tout cas comme une hypocrite dont l'intérieur et l'extérieur ne correspondent en aucune manière. Au-dehors, elle n'est que vertu ostentatoire. Au-dedans, elle est saisie d'une fièvre lubrique : «these angry hearts that break out at the lips of these strait-laced ladies are but as symptoms of a lustful fever that boils within them» (II.3.25-7).

De même, Tharsalio couvre d'injures son rival, Rebus, et ses amis, courtisans bouffis d'orgueil, tels des cornemuses : «whoreson bagpipe lords» (I.2.112). Rebus lui-même est comparé à des tumeurs diverses et abcès en tous genres, "A tumour, a imposthume, he is, madam, a very hautboy, a bagpipe, in whom there is nothing but wind, and that none of the sweetest either» (1.2.128-30). Ce vide est camouflé par une enveloppe illusoire, que Tharsalio assimile ici à de la graisse recouvrant un corps maigre : «A lean lord, dubbed with the lard of others» (I.2.115-6) ${ }^{10}$.

Mais Tharsalio lui-même joue sur l'apparence et la substance, lorsqu'il feint de n'avoir pas réussi à séduire la comtesse, et se présente à son frère et à sa belle-sœur dans un habit négligé : «What, wrapped in careless cloak, face hid in hat unbanded !» (III.1.38-9), lui dit son frère. Néanmoins, Tharsalio joue sur l'effet de surprise créé lorsqu'il révèle, sous son habit miteux, un splendide costume, et qu'il annonce son mariage avec Eudora.

Tharsalio semble être le seul à pouvoir mettre au jour les contradictions entre intérieur et extérieur. Il a acquis cette capacité durant un voyage en Italie, où ses sens se sont aiguisés, au point de percevoir, à travers la forme extérieure, la substance qui se dissimule à l'intérieur des choses et des êtres : «it hath refin'd my senses, and made me see with clear eyes, and to judge of objects as they truly are, not as they seem, and through their mask to discern the true face of things» (I.1.132-5). L'école italienne à laquelle a été formé Tharsalio, c'est bien sûr celle de Machiavel, qui lui a révélé les secrets des apparences : «Tout le monde voit bien ce que tu sembles, mais bien peu ont le sentiment de ce que tu es», dit Machiavel dans Le Prince". Tharsalio est un machiavel qui utilise la dissimulation pour arriver à ses fins.

La relation entre intérieur et extérieur est primordiale dans l'œuvre dramatique de Chapman en général, ne serait-ce que dans l'opposition qu'il va décliner dans plusieurs pièces, notamment ses tragédies, entre «goodness», c'est-à-dire la vertu intrinsèque de l'homme, et «greatness», 
que l'on pourrait définir comme une grandeur sociale qui n'est qu'apparence. Ainsi, dans Bussy D'Ambois (1607), Chapman dénonce les courtisans qui n'ont que l'apparence de la grandeur. Au-dedans, ils ne sont que vide :

Think they bear all the kingdom's worth before them ;

Yet differ not from those colossic statues,

Which with heroic forms without o'erspread,

Within are nought but mortar, flint and lead.

(Bussy D'Ambois, I.1.14-7)

Le thème du contenant vide, dont la variante, le sépulcre vide, domine The Widow's Tears, semble donc être un des fondements de l'idéologie chapmanienne. Pour Chapman, il y a toujours déchéance de la substance au profit de la forme, et donc «insubstantialité» fondamentale de l'homme : «Man is a torch borne in the wind; a dream / But of a shadow, summ'd with all his substance» (Bussy D'Ambois, I.1.5-19). L'image s'inscrit dans une vision nostalgique du monde, selon laquelle le poète considère que l'équivalence entre intérieur et extérieur a existé dans un passé idéalisé, mais qu'elle n'est plus possible en ce monde. Dans les pièces et les poèmes, on trouvera donc abondance de ce que l'on appelait en français du XVIe siècle des «vidures», c'est-à-dire des motifs en creux - bulles, outres, tumeurs, ventres gonflés d'air, etc. - espaces vides pourvus d'un extérieur dont le but est de tromper le regard ${ }^{12}$.

La pièce de Shakespeare, Pericles ${ }^{13}$, probablement composée en 1607 et déposée au Registre des libraires en 1608, s'articule également autour d'une multitude d'apparences illusoires et de contenants détournés de leur fonction originelle, voire, dans le pire des cas, vides de tout contenu. Cette pièce composite intrigue tant par le mystère de son écriture que par sa construction dramatique inhabituelle qui épouse la fuite du héros éponyme d'Antioche à Mytilène en passant successivement par Tyr, Tharse, Pentapolis, et Éphèse. Certes, les cinq interventions de John Gower, le poète ami de Chaucer qui écrivit l'histoire d'Apollonios de Tyr dans Confessio amantis (1380-1390), source principale de Pericles, cadrent la pièce et lui assurent une certaine cohésion structurelle en reliant les différentes étapes de l'errance de Périclès. Mais c'est surtout la thématique de la disjonction entre essence et apparence, entre contenu et contenant qui confère à la pièce un fil directeur et une unité qui sinon lui feraient défaut. 
La première scène introduit d'emblée le thème du questionnement des apparences, puisque Périclès doit percer l'énigme que lui propose Antiochus afin d'obtenir la main de sa fille. Lors du prologue, Gower avait souligné la beauté physique hors du commun de la princesse - «So buxom, blithe and full of face / As heaven had lent her all his grace» (chœur, acte 1.23-4). Mais sous le masque séduisant de la beauté se dissimule la relation incestueuse entre le père et la fille, ainsi que le découvre Périclès, qui assimile alors la princesse à un coffret au contenu corrompu : «Fair glass of light, I lov'd you, and could still, / Were not this casket stor'd with ill» (I.1.77-8). Cette métaphore du coffret trompeur fait écho au coffret doré, bien réel celui-là, à l'intérieur duquel se trouvait un crâne dans The Merchant of Venice ${ }^{14}$. Dans les deux cas, Shakespeare se livre à une inversion du motif platonicien du silène, cet objet à l'extérieur ridicule et au contenu précieux, tel qu'on le trouve décrit par Alcibiade dans Le Banquet :

[...] ces silènes qu'on voit exposés dans les ateliers des sculpteurs, et que les artistes représentent un pipeau ou une flûte à la main; si on les ouvre en deux, on voit qu'ils contiennent, à l'intérieur, des statues de dieux."

Alcibiade a recours au silène pour faire l'éloge de son ami Sọcrate dont l'aspect extérieur difforme cache une substance rare :

D'un autre côté, il ignore tout, il ne sait rien c'est du moins l'air qu'il se donne. N'est-ce point la façon d'un silène? $\mathrm{Si}$, tout à fait, car tels sont les dehors du personnage, à la manière du silène sculpté. Mais le dedans ? Une fois le silène ouvert, avez-vous idée de toute la sagesse dont il regorge, ô buveurs mes amis ?

Le motif platonicien du silène se répand dans toute l'Europe à la Renaissance grâce à Érasme, pour qui il est une figure de prédilection. Ce dernier y fait référence dans L'Éloge de la folie, dans l'Enchiridion, et lui consacre un essai complet intitulé «Sileni Alcibiadis» qui paraît pour la première fois dans l'édition en latin de 1515 des Adages, œuvre qui connaîtra de multiples rééditions au cours du XVIe siècle tant en latin qu'en langues vernaculaires : 
[...] les Silènes étaient des figurines fendues d'une manière telle qu'on pouvait séparer les deux parties et ouvrir la figurine; fermées elles ne présentaient qu'une apparence risible et déformée de joueur de flûte, mais ouvertes elles montraient soudain une divinité, de telle sorte que la plaisante tromperie rendait plus agréable l'art du sculpteur.

L'originalité d'Érasme dans cet adage réside surtout dans son appropriation du motif du silène et dans sa création d'un nouveau paradigme, le silène inversé, «Inversorum Silenorum», selon lui beaucoup plus répandu dans le monde que le silène originel décrit par Alcibiade :

\footnotetext{
Aujourd'hui encore il y a, bien cachés, quelques bons Silènes, mais hélas trop peu nombreux. La majorité des hommes ressemblent à un Silène inversé. Si l'on observait jusqu'en leur fond le sens et la nature des choses, on trouverait que personne n'est plus éloigné de la vraie sagesse que ceux qui avec des titres magnifiques, des bonnets pleins de sagesse, des ceintures splendides, des anneaux portant pierres précieuses, professent une sagesse accomplie.
}

À l'instar de Bassanio, dans The Merchant of Venice, qui contemple la boîte et déjoue les apparences trompeuses, «So may the outward shows be least themselves. / The world is still deceived with ornament» (III.2.73-4), Périclès décèlera chez le père comme chez la fille le vice masqué par la beauté :

How courtesy would seem to cover sin,

When what is done is like an hypocrite,

The which is good in nothing but in sight !

(I.1.122-4)

Le motif du silène inversé réapparaît à l'acte I, scène 4 , lorsque Cléon, à l'approche des voiles blanches de la flotte de Périclès, exprime sa méfiance des belles apparences: "Who makes the fairest show means most deceit» (I.4.75). Périclès, dont l'ambition est de faire coïncider apparence et essence, l'assure alors de son honnêteté, et ce que Cléon 
pensait être une menace de mort se révèle être son salut : «And these our ships, you happily may think / Are like the Trojan horse was stuff'd within / With bloody veins expecting overthrow, / Are stor'd with corn to make your needy. bread» (I.4.91-5). Le Cheval de Troie, auquel fait allusion Périclès, peut être lu comme un avatar du silène inversé. En effet, les Troyens sont victimes de l'apparence extérieure de l'objet qui contient en son sein la destruction de la ville, ainsi que le chante l'aède Démodocos dans le chant VIII de l'Odyssée, traduite par Chapman et publiée en 1614:

To bring the Fate and death they after did.

He sung, besides, the Greeks' eruption

From those their hollow crafts, and horse foregone ;

And how they made Depopulation tred

Beneath their feete so high a Citie's head."

À Tharse comme précédemment à Antioche, Périclès affirme son refus de l'objet trompeur et mortifère, du silène inversé. En rejetant l'assimilation de ses vaisseaux avec le Cheval de Troie, en remplaçant la menace de mort par une promesse de vie, «And give them life whom hunger starv'd half dead» (I.4.96), il tente de réconcilier l'intérieur et l'extérieur, l'apparence et la substance. Au cours de son errance, Périclès sera progressivement amené à se rapprocher du silène tel que le définit Platon, cachant son rang et son titre derrière une apparence de dénuement, avant de finalement retrouver son identité de père, d'époux et de prince.

Le thème de la confusion des apparences est repris, sur un mode comique, à l'acte II, lorsque, dès la première scène, les pêcheurs découvrent l'armure rouillée de Périclès qu'ils confondent initialement avec un poisson. Dans la scène 2 de l'acte II, écho déformé de la scène 1 de l'acte I, Périclès se rend à Pentapolis pour prendre part au tournoi dont le vainqueur obtiendra la main de Thaisa, fille du roi Simonidès. On assiste alors à la victoire de Périclès-silène, dont Simonidès comme Thaisa ont perçu la véritable noblesse sous la misérable armure rouillée qui faisait se gausser les autres chevaliers. À l'inverse d'Antiochus, Simonidès apparaît comme un fidèle disciple de Platọn, condamnant le monde des apparences au profit d'une substance cachée :

Opinion's but a fool, that makes us scan The outward habit by the inward man. 
On peut également lire dans le défilé des six chevaliers présentant chacun leur devise à Thaisa, afin qu'elle soit glosée par Simonidès, une série de variations autour du silène. En effet, ainsi que le rappelle Edgar Wind, emblèmes et devises, comme les silènes, étaient prisés pour leur obscurité et leur capacité à suggérer une vérité tout en la dissimulant ${ }^{20}$. Parmi ces écus, celui du quatrième chevalier représentant une torche brûlant à l'envers, accompagné de la devise "Qui me alit, me extinguit» ${ }^{2 !}$ souligne la dangereuse réversibilité des choses et du monde, et rappelle l'instabilité ontologique constitutive du silène. Cette devise annonce la confusion centrale de la pièce entre la mort et la vie, pour Thaisa comme pour Marina.

En effet, grâce à l'art du mage Cérimon, lui même défini comme un véritable silène privilégiant la richesse intérieure aux apparences trompeuses, «I hold it ever, / Virtue and noble cunning were endowments greater / Than nobleness and riches» (III.2.26-8), le cercueil de Thaisa, supposé contenir la mort, abritera finalement la vie : «I op'd the coffin, I Found there rich jewels ; recover'd her, and plac'd her / Here in Diana's temple» (V.3.23-5). Le faux cercueil de Thaisa, interface réversible entre la vie et la mort, offre une variation supplémentaire sur le motif du silène tel que le définissait Érasme dans L'Éloge de la folie :

D'abord, c'est un fait que toutes les choses . humaines à la manière des silènes d'Alcibiade, ont deux faces tout à fait différentes. Ainsi ce qui à première vue est la mort, est la vie si vous regardez plus à l'intérieur des choses. Ce qui était la vie, est la mort ; ce qui était beau, laid; l'opulence cache l'indigence; l'infamie, la gloire; le savoir, l'ignorance; la force, faiblesse; la noblesse, l'obscurité ; la joie, chagrin ; la prospérité, disgrâce ; l'amitié, inimitié ; le remède, le poison ; bref, si vous ouvrez le silène vous verrez soudain tout inversé. ${ }^{22}$

À la fausse mort de Thaisa fait écho la description par Dionysa du tombeau vide, car dépourvu de cadavre, de Marina. Ce contenant sans contenu constitue la mise en cause ontologique la plus radicale. Il ne s'agit plus seulement d'opposition entre extérieur et intérieur mais de la négation même de la fonction de contenant. Dionysa, ayant ordonné à Léonine le meurtre de Marina - forfait qu'il n'a pas accompli, même s'il a affirmé 
avoir jeté le corps à la mer - tente de convaincre son époux Cléon que la magnificence du tombeau suffira à endormir les soupçons de Périclès :

Her monument

Is almost finish'd, and her epithaphs

In glitt'ring golden characters express

A general praise to her, and care in us

At whose expense 'tis done.

(IV.3.42-6)

Ce tombeau richement orné, «in glitt'ring golden characters», n'est pas sans rappeler cet autre contenant trompeur, le coffret auquel Périclès comparait la princesse d'Antioche à l'acte $\mathrm{I}$, scène 1. Dionysa, habile dans la manipulation des apparences, pense pouvoir dissimuler sa trahison et la bassesse de son crime derrière la richesse du sépulcre, mais par un renversement ironique, elle sera à son tour victime des apparences qu'elle croyait maîtriser ; en effet seul le spectateur sait que Marina, enlevée par des pirates a survécu, et que le tombeau n'est qu'une enveloppe vide. Si le cercueil de Thaisa dissimule non pas un cadavre mais un corps vivant, le monument érigé pour Marina et censé contenir la mort ne contient absolument rien. En outre, ce monument funéraire vide s'oppose à la statue élevée à la mémoire de Périclès qui a sauvé Tharse de la famine, ainsi que le rappelle Gower dans le prologue de l'acte II : «And, to

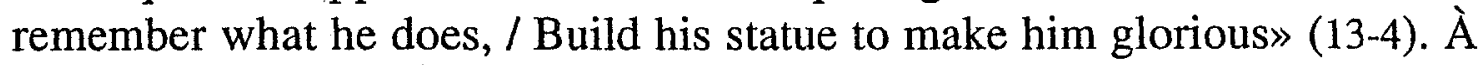
l'inverse du tombeau vide de Marina, contenant trompeur, la statue de Périclès, célébration aux yeux de tous de la noblesse du personnage, suggère la possibilité d'une adéquation de la substance et de l'apparence. Toutefois, on notera que contrairement au sépulcre vide dans The Widow's Tears, symbole de la fausseté qui prévalait dans l'ensemble de la pièce de Chapman, dans Pericles, le tombeau sans cadavre est le garant de la vie préservée de Marina. Dionysa, quant à elle, fournirait une nouvelle version du silène inversé, comme le souligne Cléon lorsqu'il la compare à une harpie : «Thou art like the harpy, / Which, to betray, dost with thine angel face, / Seize with thine eagle's talons» (IV.3.46-8). Peut-être faut-il voir dans le nom même de cette harpie, Dionysa, une référence paradoxale et ironique à Dionysos, élève supposé du satyre prénommé Silène ${ }^{23}$ ?

À l'opposé de Dionysa, Marina est censée incarner une correspondance retrouvée entre ce qui paraît et ce qui est. En effet, lorsqu'elle se trouve confrontée à Périclès dans la scène 1 de l'acte $\mathrm{V}$, elle 
se réclame d'une transparence parfaite : «Yet I was mortally brought forth, and am / No other than I appear» (V.1.104-5). C'est également ainsi que la percevra son père, qui voit en elle l'incarnation de la vérité et l'adéquation entre apparence et essence :

Falseness cannot come from thee, for thou look'st Modest as Justice, and thou seem'st a palace For the crown'd Truth to dwell in.

Pourtant, lorsqu'elle se retrouve, après son enlèvement par des pirates auxquels elle doit la vie, dans un bordel de Mytilène à enseigner à ses clients la chasteté et la valeur morale, cachant sa vertu sous le masque de la prostitution, elle se rapproche paradoxalement davantage du silène. Celle qui affirmait à tort faire confiance aux dehors et ne se méfiait pas du visage agréable de Léonine, "You are well favour'd, and your looks foreshow / You have a gentle heart» (IV.1.85-6), se révèle capable de manipuler les apparences au point de transformer une maison close en un lieu de prêche : «But to have divinity preach'd there! Did you ever dream of such a thing ?» (IV.5.4-5), s'étonneront, en la quittant, quelques clients convertis.

Dans Pericles, l'utilisation faite par Shakespeare du silène et de ses avatars est particulièrement originale car contradictoire ${ }^{24}$; la figure du silène platonicien - qu'il s'agisse principalement de Périclès, de Cérimon, ou de Marina - alterne avec celle du silène inversé érasmien Antiochus et sa fille, Cléon et Dionysa - voire avec la possibilité, certes limitée, d'une correspondance idéale entre apparence et essence. De plus, un même personnage - c'est le cas de Marina et de Périclès - peut prôner l'adéquation de l'apparence et de la substance tout en se conformant aux caractéristiques du silène platonicien. Enfin, le dénouement de la pièce s'inscrit également dans cette logique contradictoire : avant que la vérité triomphe sur l'illusion pour conduire à la reconnaissance finale, le héros éponyme n'a cessé d'être le jouet des apparences, au point de ne pouvoir distinguer entre la vie et la mort. L'oscillation constante entre silène et silène inversé témoignerait chez le dramaturge d'une volonté de confusion des repères. Le silène, par ses 
possibilités de jeu sur la notion d'interface, serait pour Shakespeare un motif privilégié au service d'une esthétique de la réversibilité.

Au contraire, le silène a pour Chapman une valeur idéologique. La rupture entre intérieur et extérieur est pour lui l'indice d'une corruption irréversible du monde. Il n'y a pas, chez lui, de réversibilité possible, car elle est toujours limitée, butant irrémédiablement sur la vacuité. Mais c'est surtout dans la vision qu'il a de son œuvre poétique dans son ensemble qu'il exploite le décalage entre le contenant et le contenu. Chapman considère son œuvre comme un contenant dont le contenu n'est pas visible aux yeux de tous. Élitiste il l'est, lorsqu'il affirme, dans sa dédicace de Ovids Banquet of Sence (1595) à Mathew Roydon, que son œuvre ne s'adresse qu'à ceux qui ont atteint à la véritable noblesse, c'est-à-dire qui sont détenteurs d'une véritable substance: «The prophane multitude I hate, \& onelie consecrate my strange Poems to these serching spirits, whom learning hath made noble, and nobilitie sacred» ${ }^{25}$. On notera qu'il s'agit de ne pas s'arrêter à la surface du contenant («my strange Poems»), mais d'aller chercher le contenu là où il se trouve, au fond des choses, au fond de la bôtte. On retrouve d'ailleurs presque la même terminologie dans l'adage d'Érasme «Les Silènes d'Alcibiade»: «De même dans la connaissance authentique la vérité est toujours ce qui est le plus profondément caché et elle n'est pas saisie facilement ni par le grand nombre» ${ }^{26}$. On pourrait voir une correspondance entre le motif du silène et l'abondance d'images mettant en évidence, dans la poésie et le paratexte chapmaniens, l'opposition entre intérieur et extérieur. Chapman, grand lecteur des Adages, comme nombre de ses contemporains, va d'ailleurs faire appel à une image bien connue qu'il a sans doute trouvée dans «Les Silènes d'Alcibiade»: «La nature a renfermé l'or et les pierres précieuses dans les plus profondes cachettes de la terre» ${ }^{27}$. Chapman l'utilise pour montrer que, dans la représentation poétique, l'étrangeté n'est qu'extérieure, et que l'obscurité est nécessaire à la révélation de pierres précieuses, dissimulées dans le fond de ce silène :

Obscuritie in affection of words, \& indigested concets, is pedanticall and childish; but where it shroudeth it selfe in the hart of his subject, utterd with fitnes of figure, and expressive Epithetes ; with that darknes wil I still labour to be shaddowed; rich Minerals are digd out of the bowels of the earth, not found in the superficies and dust of it. ${ }^{28}$ 
Le poète doit être une sorte de prêtre révélant les mystères de cette poésie, «[the] misteries and allegoricall fictions of Poesie» ${ }^{20}$. Néanmoins, seuls les initiés qui possèdent le vrai savoir peuvent y avoir accès. Il ne faut donc jamais se fier aux apparences, toujours trompeuses, que le contenant soit vide ou qu'il soit plein. Pour Chapman, comme il l'explique dans un de ses commentaires de sa traduction de l'Iliade, l'obscurité du style est le garant d'une substance riche - vrai savoir, donc vraie noblesse, pour lui - se dissimulant au cœur de la poésie, et s'opposant au «plain style», qui n'a de poésie que les contours, et pas de substance :

[...] this case is ruled against such men, that they affirme these hypertheticall or superlative sort of expressions and illustrations are too bold and bumbasted; and out of that word is spunne that which they call our Fustian, their plain writing being stuffe nothing so substantial but such grosse sowtedge, or hairepatch, as every goose may eate through.

Il ne faut donc pas s'arrêter à la surface de cette écriture enveloppée de tissu épais et travaillé - la futaine - car, contrairement à ces étoffes légères comme la haire, elle dissimule des trésors.

Il n'est peut-être pas fortuit que Chapman nous ait laissé des écrits théoriques sur son œuvre, puisque l'idéologue qu'il est la voit comme figée dans une signification particulière, traversée par une cassure irrémédiable entre l'intérieur et l'extérieur. De Shakespeare, au contraire, il ne nous reste que la mouvance perpétuelle du motif, dans laquelle la cassure même peut se voir remise en question à l'infini.

Laetitia COUSSEMENT-BOILLOT Université Paris VII - Denis Diderot / Christine SUKIC Université de Bourgogne 


\section{NOTES}

'On en trouyera un exemple caractéristique dans les mystères qui étaient joués à Chester. La scène de la Résurrection est particulièrement longue dans ce cycle de pièces, et elle met l'accent sur la présence du sépulcre vide, qui provoque les lamentations de Marie Madeleine :

Ah, Peter and John, alas, alas !

There is befallen a wondrous case.

Some man my Lord stolen has

And put him I wot not where.

(The Chester Mystery Cycle. A New Edition with Modernised Spelling, éd. David Mills, East Lansing (Michigan), Colleagues Press Inc., 1992, p. 329). David Mills, dans son introduction à la scène, montre d'ailleurs l'importance du sépulcre dans ce passage: «The focus of the action is obviously the sepulchre which, from contemporary art and from the disposition of the soldiers, seems to have been a table-tomb whose flat top slab lifts or slides to allow Christ to stand and step out on to one of the soldiers [...]. The empty tomb, with Peter kneeling before Jesus, forms the final tableau» (ibid., p. 317).

${ }^{2}$ Sur les sépulcres dans les églises anglaises du Moyen Âge, voir Neil C. Brooks, The Sepulchre of Christ in Art and Liturgy, with special reference to the Liturgic Drama, University of Illinois Studies in Language and Literature, Urbana, University of Urbana Press, 1921, p. 71-91.

${ }^{3}$ Comme le montre Solange Corbin, le sépulcre est «le seul [élément] dans toute la déposition, qui évoque le théâtre» (La Déposition liturgique du Christ au vendredi saint. Sa place dans l'histoire des rites et du théâtre religieux, Paris, Les Belles Lettres, Lisbonne, Livraria Bertrand, 1960, p. 197).

${ }^{4}$ Par exemple, dans Psyche, or, Loves Mysterie de Joseph Beaumont (1648), les femmes se lamentent en découvrant le tombeau : «Dear Sirs, O what, alas what shall we do ! / The only relict of our Hopes is gone» (strophe 138, vers 829-30). Mais bien vite un ange les détrompe : «He told us that our Savior from his Bed / Of death was risen» (Canto XV, strophe 139, vers 837-8).

${ }^{5}$ Pour une discussion de la date de composition de la pièce, voir The Widow's Tears, éd. Akihiro Yamada, The Revels Plays, Londres, Methuen, 1975, p. xxxi-xxxiii.

${ }^{6}$ L'édition utilisée ici est celle de Ethel M. Smeak, Regents Renaissance Drama, Lincoln, University of Nebraska Press, 1966.

'Ibid., p. Ixvii-lxxii. Voir également Alan C. Dessen, Recovering Shakespeare's Theatrical Vocabulary, Cambridge University Press, 1995, p. 1823. 
${ }^{8}$ On trouve d'ailleurs la même expression dans son poème, The Teares of Peace (publié en 1609), lorsque la Paix pleure devant le cercueil de l'amour humain. La vanité humaine y est dénoncée par l'image d'un sépulcre: «This breathing Sepulcher» (vers 1019, in Plays and Poems, éd. Jonathan Hudston, Renaissance Dramatists, Harmondsworth, Penguin Books, 1998, p. 318).

'En ce qui concerne Chapman, Franck Schoell pense que cette image a pour origine l'adage d'Érasme vivum cadaver, vivum sepulchrum (Études sur l'humanisme continental en Angleterre à la fin de la Renaissance, Paris, Librairie Ancienne Honoré Champion, 1926, p. 53-4).

${ }^{10}$ On peut aussi voir dans cette dénonciation une allusion aux chevaliers écossais créés par Jacques Ier dans les premières années de son règne, et dont Chapman s'était déjà moqué dans Eastward Ho !, pièce écrite en collaboration avec Ben Jonson et John Marston, et publiée en 1605. Comme on le sait, ces allusions valurent aux trois poètes d'être jetés en prison pour outrage à leur souverain.

"Machiavel, Euvres complètes, éd. Edmond Barincou, Bibliothèque de la Pléiade, Paris, Gallimard, 1952, p. 343.

${ }^{12}$ Ainsi, dans The Revenge of Bussy D'Ambois, les politiciens sont comparés à des boursouflures ou des excroissances : "They are puft / With such proud tumours» (I.1.308-9). Dans la même pièce, et avec le même sens, on note aussi l'emploi du mot «emrods» (I.1.267), qui désigne une tumeur hémorroïdale.

${ }^{13}$ Pericles est souvent négligée par la critique car considérée comme le fruit d'une collaboration entre Shakespeare, à qui on attribue au moins les trois derniers actes, et d'autres dramaturges mineurs. Parmi les co-auteurs possibles, George Wilkins et John Day sont les plus fréquemment cités, même si l'on ne dispose d'aucune certitude à leur égard. L'édition utilisée dans cette étude est celle établie par F. D. Hoeniger, The Arden Shakespeare, 2000.

${ }^{14}$ Voir II.7.65-9.

${ }^{15}$ Platon, Le Banquet, 215 b, Euvres Complètes, tome IV, traduction de Paul Vicaire, Paris, Les Belles Lettres, 1992, 2ème édition revue et corrigée.

${ }^{16}$ Platon, Le Banquet, op. cit., 216 d-e.

"Érasme, «Les Silènes d'Alcibiade», Les Adages, traduction de Jacques Chomarat, Euvres choisies, Paris, Poche, 1991, p. 402. Certaines traductions anglaises anonymes de l'époque étant peu fiables, on a choisi de citer Érasme en français dans le cadre de cette étude.

${ }^{18}$ Érasme, ibid., p. 408.

${ }^{19}$ Chapman's Homer, The Odyssey, The Eighth Booke, 1.678-97, ed. Allardyce Nicoll, Princeton et Oxford, Princeton U.P., 2000, p. 146.

${ }^{20}$ Edgar Wind, Mystères païens de la Renaissance, trad. Pierre-Emmanuel Dauzat, Paris, Gallimard, 1992, p. 178-9. Titre original, Pagan Mysteries in the Renaissance, Londres, Faber and Faber Limited, 1958. 
${ }^{21}$ «Who feeds me extinguishes me».

${ }^{22}$ Érasme, L'Éloge de la folie, traduction du latin de Claude Blum, ParisGenève, Éditions Slatkine, 1995, chap. XXIX, p. 91-2.

${ }^{23}$ Dans la mythologie grecque, le silène est le «nom générique des satyres devenus vieux», mais également le nom d'un personnage, doué d'une grande sagesse, fils de Pan ou d'Hermès, qui passait pour avoir élevé Dionysos. Cf. Pierre Grimal, Dictionnaire de la mythologie grecque et romaine, Paris, P.U.F., 1996.

${ }^{24}$ Les variations autour du silène platonicien et du silène inversé érasmien apparaissent tôt dans l'œuvre de Shakespeare, dans ses comédies comme dans ses tragédies. Déjà dans Romeo and Juliet, on repère un silène inversé dans la description que fait Juliette de son amant lorsqu'elle apprend le meurtre de Tybalt. Derrière le portrait oxymorique bien connu, «beautiful tyrant, fiend angelical...», opposant la beauté physique de Roméo à la laideur de son acte, se dessine l'opposition d'un contenant séduisant masquant un contenu dégradé : « $\mathrm{O}$, that deceit should dwell / In such a gorgeous palace !» (III.2.36-84).

${ }^{25}$ George Chapman, Plays and Poems, op. cit., p. 239.

${ }^{26}$ Érasme, «Les Silènes d'Alcibiade», op. cit., p. 411.

${ }^{27}$ Érasme, ibid., p. 409.

${ }^{28}$ Plays and Poems, op. cit., p. 239-40.

${ }^{29}$ A Free and Offenceles Justification, Of A Lately Publisht and most maliciously misinterpreted Poeme ; Entituled Andromeda Liberata, Londres, 1614, cité dans Plays and Poems, ibid., p. 381.

${ }^{30}$ Chapman's Homer. The Iliad, éd. Allardyce Nicoll, Bollingen Series XLI, Princeton (N. J.), Princeton University Press, «The Fourteenth Book. Commentarius», p. 295. 